\title{
Near-Earth asteroids among the Scorpiids meteoroid complex (Research Note)
}

\author{
P. B. Babadzhanov ${ }^{1}$, I. P. Williams ${ }^{2}$, and G. I. Kokhirova ${ }^{1}$ \\ 1 Institute of Astrophysics, Tajik Academy of Sciences, 734042 Dushanbe, Tajikistan \\ e-mail: Kokhirova2004@mail.ru \\ 2 Queen Mary University of London, E1 4NS, UK \\ e-mail: I.P.Williams@qmul .ac.uk
}

Received 3 April 2013 / Accepted 20 June 2013

\begin{abstract}
A group of near-Earth asteroids (NEAs) that moved on similar orbits to each other and where the orbits could be classed as cometary were identified by searching the "Near-Earth Objects Dynamic Site" database. Six NEAs were identified, 2003HP2, 2006WX29, 2007VH189, 2007WT3, 2007WY3, and 2008UM1. The orbits were integrated back over one complete cycle in the variation of the argument of perihelion to identify times when their nodal distance was $1 \mathrm{AU}$. Theoretical meteoroids were assumed to have been released at these times on an orbit identical to those of the NEAs. The characteristics of a meteor shower that would be formed when these meteoroids hit the Earth's atmosphere were calculated. It was found that the showers produced from all six NEAs were identical and could be recognized as the nighttime $\chi$ - and $\delta$-Scorpiids and the daytime $\beta$ - and $\sigma$-Librids, the latter two being named by us. It was also found that the orbital evolution of the Scorpiids was almost identical to the orbital evolution of the six NEAs. The similarity of the orbits, the nature of the orbit as cometary and the association of the Scorpiid meteoroid stream lead us to suggest that the origin of the whole complex was the fragmentation of some unknown comet several millennia ago.
\end{abstract}

Key words. meteorites, meteors, meteoroids - minor planets, asteroids: general

\section{Introduction}

The inner solar system is populated by asteroids, comets and meteoroids as well as the terrestrial planets. Though the boundary is not specifically defined, all bodies with a size from several tens of microns to several meters are called meteoroids, smaller objects are regarded as dust grains and larger ones as comets or asteroids. The main source of meteoroids is thought to be comets and asteroids formed through outgassing fragmentation and collisions. If a meteoroid enters the Earth's atmosphere, a meteor will be produced as it ablates, which can be studied from the ground using several techniques. The majority of the observed meteors appear randomly, both in time and position, and are called the sporadic background. A meteor shower is an occurrence when the number of observed meteors is significantly higher than the general background and where these meteors have a well-defined radiant on the sky. This implies that they all have similar heliocentric orbits, and following from that, that they originate from a meteoroid stream whose orbital elements can be determined.

Initially it was believed that meteoroid streams originated in comets. This connection was confirmed with the observed disintegration of comet 3D/Biela and the associated Andromedids meteor shower (recently discussed again by Jenniskens \& Vaubaillon 2007). The notion that meteoroid streams might be associated with asteroids is also quite old (see Williams 2011, for a historical review of this topic and relevant references).

As seen from Earth, comets differ from asteroids because they show evidence of mass ejection in the form of a coma and tail. This difference is a consequence of their different physical natures. Comet nuclei are composed primarily of ices with some embedded solid particles, but asteroids are formed out of stony or iron material with little or no evidence of ices. This physical difference arises because comets formed in the outer regions of the solar system while asteroids formed inside the orbit of Jupiter. This leads to a general difference between the orbits of comets and asteroids and several criteria have been developed to distinguish between an asteroidal and a cometary orbit. These have recently been reviewed by Jopek \& Williams (2013), and one of the most widely used is based on the Tisserand invariant

$T_{\mathrm{j}}=\frac{a_{\mathrm{j}}}{a}+2\left[\frac{a}{a_{\mathrm{j}}}\left(1-e^{2}\right)\right]^{0.5} \cos i$,

where $a, e$, and $i$ are the semi-major axis, eccentricity, and inclination of the object's orbit, respectively, and $a_{\mathrm{j}}=5.2 \mathrm{AU}$ is the semi-major axis of the orbit of Jupiter. Kresak (1969) suggested that for comets $2.08<T_{\mathrm{j}} \leq 3.12$, and for asteroids $T_{\mathrm{j}}>3.12$. Since that time, the limits have been slightly modified, but a value of $T_{\mathrm{j}}$ of about 3 is still considered to be the boundary between asteroidal and cometary orbits (Jewitt 2012).

Recent discoveries have shown that the distinction between asteroids and comets is not quite as clear-cut. For example, asteroids can occasionally release dust and form a short-lived tail, as was seen in the image of 2010A2 (Linear). This was caused by a collision in February or March 2009 (Jewitt et al. 2010; Snodgrass 2010). Conversely, there is a small group of objects that are moving on orbits within the main asteroid belt with a Tisserand invariant $T_{\mathrm{j}}$ well in excess of 3 , but show cometary activity. These were called main-belt comets by Hsieh \& Jewitt (2006). 
Asteroids with perihelion distances shorter than 1.3 AU are called near-Earth asteroids (NEAs) for obvious reasons. To date, more than 9000 such asteroids have been discovered ${ }^{1}$.

A significant fraction of this NEA population were removed from the main asteroid belt through the effects of the gravitational fields of the major planets, mainly Jupiter (Farinella et al. 1992). However, some of the NEAs may be dead or dormant cometary nuclei from the Jupiter family, where a thick dust mantle prevents the sublimation of volatiles under solar heating (Jewitt 2012), or they could remnants following the fragmentation of the parent comet.

Determining the geometric albedo is in principle a way of distinguishing between a dormant comet and an asteroid, but obtaing this requires simultaneous observations in both the visible and the infra-red to obtain both the reflected and re-emitted part of the emission. This has only been achieved for a tiny number of NEAs (Fernandez et al. 2001).

An alternative approach is to consider meteoroid streams. As already mentioned, collisions between asteroids can generate a meteoroid ejection. However, Williams (1993) claimed that streams formed in this way would contain far less mass and be far more diffuse than those from a cometary origin. Comets eject meteoroids at every perihelion passage and so can produce a much stronger stream. Hence, the existence of meteor showers associated with some NEAs is strong evidence that these asteroids are dormant comets or fragments of the original nucleus (Olsson-Steel 1988; Babadzhanov 1996; Jenniskens 2004). The meteoroids will initially move on orbits close to that of the comet at that time. As the cometary orbit evolves, meteoroids will also be found that match the cometary orbit at these times. Sometimes, a particular meteor outburst can be traced back to a specific perihelion passage of the comet, as was first done by Asher et al. (1999), showing that the Leonid meteors observed in 1998 were ejected from comet 55P/Tempel-Tuttle in AD1333. More often the streamlets formed at each perihelion passage merge into a broader overall stream that still shows evidence of the earlier ejections.

It is often the case that comets fragment towards the end of their life, giving rise to several NEAs with orbits that are similar to both the parent comet at that time and the meteoroid stream. The Taurid complex is a good example, where many asteroids as well as comet $2 \mathrm{P} /$ Encke move within the meteoroid complex (Asher et al. 1993; Babadzhanov 2001; Porubčan et al. 2004, 2006; Babadzhanov et al. 2008a). Another example is the Quadrantid stream, which is related to comet Machholtz, $2003 \mathrm{EH} 1$, and possibly comet 1481 (Jenniskens 2004; Williams et al. 2004; Neslusan et al. 2013). The similarity between the Geminids and asteroid 3200 Phaethon was first noticed by Whipple (1983). Asteroids 2005UD and 1999YC also have similar orbits to both Phaethon and the Geminids (Ohtsuka et al. 2006; Jewitt \& Hsieh 2006; Kinoshita 2007). Several works have pointed out that Phaethon had all the characteristics necessary to be the parent of the Geminid meteoroid stream assuming that the ejection mechanism takes place continuously over a wide range of true anomaly (Fox et al. 1983; Hunt et al. 1985; Williams \& Wu 1993; Ryabova 2007). Hence, in this case, the comet has become dormant or totally disintegrated. The Piscids meteoroid stream has three associated NEAs (Babadzhanov \& Williams 2007; Babadzhanov et al. 2008b), while four NEAs are related to the $\iota$-Aquariids meteoroid stream, (Babadzhanov et al. 2009). NEA 2004CK39, moving on a comet-like orbit, is associated with the $v$-Virginids meteoroid

\footnotetext{
1 http://newton.dm.unipi.it/neodys
}

stream (Babadzhanov et al. 2012). The $\chi$-Orionids are associated with asteroid 2008XM1 (Madiedo et al. 2013). Other associations have been discussed by Jenniskens \& Vaubaillon (2008) and Trigo-Rodriguez et al. (2007, 2008a,b).

Historically, an association between a meteoroid stream and an NEA has been claimed when the orbits are similar, based on criteria such as $D_{\mathrm{S}-\mathrm{H}}$, proposed by Southworth \& Hawkins (1963), that is

$$
\begin{aligned}
D_{\mathrm{S}-\mathrm{H}}^{2}= & \left(e_{2}-e_{1}\right)^{2}+\left(q_{2}-q_{1}\right)^{2}+\left(2 \sin \frac{i_{2}-i_{1}}{2}\right)^{2} \\
& +\sin i_{1} \sin i_{2}\left(2 \sin \frac{\Omega_{2}-\Omega_{1}}{2}\right)^{2} \\
& +\left[\left(\frac{e_{1}+e_{2}}{2}\right) 2 \sin \frac{\left(\Omega_{2}+\omega_{2}\right)-\left(\Omega_{1}+\omega_{1}\right)}{2}\right]^{2},
\end{aligned}
$$

using standard notation for the orbital elements, and subscripts 1 and 2 relate to the two orbits that are being compared. Most authors considered the orbits to be similar if their value of $D_{\mathrm{S}-\mathrm{H}} \leq$ 0.15 (Lindblad 1971a,b; Jenniskens 2006; Trigo-Rodriguez et al. 2007; Jopek \& Williams 2013; Madiedo et al. 2013), though in some circumstances different limits have been used.

In the inner solar system, the angular elements $\omega$ and $\Omega$ can change very rapidly, and Asher et al. (1993) proposed a simplified version, namely

$D^{2}=\left(\frac{a_{1}-a_{2}}{3}\right)^{2}+\left(e_{1}-e_{2}\right)^{2}+\left\{2 \sin \frac{i_{1}-i_{2}}{2}\right\}^{2}$,

with limits similar to those used for $D_{\mathrm{S}-\mathrm{H}}$.

The systematic monitoring of the skies, principally to identify asteroids that may present a danger to Earth, has led to a vast increase in the number of known asteroids. In turn, this has naturally led to a vast increase in the number of asteroids that are on similar orbits to the orbits of meteoroid streams, with a corresponding increase in the number of claimed associations between meteoroid streams and asteroids, especially for short-period streams that are near the ecliptic. For this reason, Porubčan et al. (2004) suggested that similarity of orbits should be maintained for a 5000-year period before a generic association could be claimed, and requiring a similarity over several thousand years before any association is claimed has now become the norm.

In this investigation, we use a different approach, where the association again has to be maintained over several thousand years, but instead of simply comparing orbits over the interval, we generate a theoretical meteoroid stream from the NEA in the past and calculate the observational characteristics of the meteor shower that it would now produce, which we compare with the characteristics of known showers. The method has been described in several papers such as Babadzhanov \& Obrubov (1992), Babadzhanov (1996, 2001, 2003), and Babadzhanov et al. (2008a,b, 2009, 2012) and is briefly outlined below.

\section{Method}

The standard process for forming meteoroid streams from comets, first proposed by Whipple (1951), is well known. Sublimating gas carries away small dust grains, resulting in large numbers of meteoroids being released with velocities that are small compared with the orbital speed of the parent, so that a stream is generated. Due to differences in the semi-major axes, the orbital periods will be different and some of the meteoroids 
Table 1. Summary of orbital and physical properties of the six NEAs.

\begin{tabular}{lcccccccccccc}
\hline \hline NEA & $\begin{array}{c}a \\
(\mathrm{AU})\end{array}$ & $e$ & $\begin{array}{c}q \\
(\mathrm{AU})\end{array}$ & & $\Omega^{\circ}$ & $\omega^{\circ}$ & $\pi^{\circ}$ & $\begin{array}{c}R_{\mathrm{a}} \\
(\mathrm{AU})\end{array}$ & $\begin{array}{c}R_{\mathrm{d}} \\
(\mathrm{AU})\end{array}$ & $T_{\mathrm{j}}$ & $H$ & $\begin{array}{c}d \\
(\mathrm{~km})\end{array}$ \\
\hline 2003HP32 & 2.7 & .78 & .60 & 3.4 & 188.1 & 155.4 & 343.5 & 3.6 & .6 & 2.8 & 19.6 & .60 \\
2006WX29 & 2.3 & .73 & .63 & 2.6 & 239.2 & 96.4 & 335.6 & 1.2 & 1.0 & 3.0 & 27.8 & .01 \\
2007VH189 & 2.6 & .72 & .72 & 5.9 & 75.5 & 250.2 & 325.7 & 1.6 & 1.0 & 3.0 & 23.3 & .11 \\
2007WT3 & 2.4 & .72 & .66 & 5.2 & 227.2 & 102.7 & 329.9 & 1.2 & 1.0 & 3.0 & 24.2 & .07 \\
2007WY3 & 2.8 & .77 & .66 & 11.9 & 286.4 & 50.6 & 337.0 & .8 & 2.2 & 2.8 & 18.3 & 1.1 \\
2008UM1 $^{*}$ & 2.5 & .70 & .76 & 5.1 & 208.9 & 114.5 & 323.4 & 1.8 & 1.0 & 3.0 & 32.1 & .002 \\
\hline
\end{tabular}

lag behind the parent, while others will be in front of it. As a result, the ejected meteoroids spread along the entire parent's orbit and form a complete loop in a comparatively short time (Hughes 1986; Williams 1995). The initial meteoroid stream is flat and thin; it is narrow close to perihelion, but is broader at aphelion. Gravitational perturbations cause the orbits to change, but the long-term overall variation is usually periodic and sinusoidal, as can be seen for example in Wu \& Williams (1992). Individual meteoroids can experience different perturbations from the parent. Hence, the rates and cycles of variations in $\omega$ and $\Omega$ will be different for different meteoroids. Therefore, the orbits of different meteoroids will be at different evolutionary stages at any given time. Thus, stream meteoroids can be found along all orbits of the parent body during its evolutionary cycle. This process considerably increases both the size of the meteoroid stream and its thickness (Babadzhanov 2001).

Meteoroids can only intersect the Earth's orbit at a nodal distance $r$ of about 1 AU. Hence for all meteoroids that form meteors,

$1 \approx r=\frac{a\left(1-e^{2}\right)}{1 \pm e \cos \omega}$, or $\cos \omega \approx \pm\left[\frac{a\left(1-e^{2}\right)-1}{e}\right]$.

There are four possible values of $\omega$ for given values of $a$ and $e$ that satisfy this condition. Thus, as the cycle of orbital evolution progresses over several thousand years, there are four different locations where a meteor shower might be formed from the meteoroid stream. At the first location, the shower could be weak because there has been significant time for the meteoroids to disperse, while the last location should produce a strong shower.

The method thus requires integrating the orbit of a given NEA back in time for a full cycle, locating the values of $\omega$ where the nodal distance is $1 \mathrm{AU}$ and calculating the orbital elements at that time, which are taken as the mean orbit of the meteoroid stream formed then. The radiant and appearance time of a meteor shower is then deduced. Finally, a search is made for actual meteor showers that agree with the theoretical predictions.

As mentioned previously, there are over 9000 known NEAs, and calculating the characteristics of meteoroid streams formed from all of these over several thousand years is a gigantic undertaking. Hence ways must be found to reduce this number.

Since the assumption is that the initial parent was a comet, we first calculated the Tisserand invariant and retained only those NEAs for which $T_{\mathrm{j}}<3.1$. Because the original comet is likely to have fragmented, we would expect several NEAs to be associated with any meteoroid stream. We have used the $D$ criterion to identify groups of NEAs within the above sub-set. Because the intention is to find such a grouping, not prove an association, at this stage a limiting value of 0.2 was used.

After identifying potential candidates, their orbital evolution was investigated using the Halphen-Goryachev integration method (Goryachev 1937), unless close encounters with Jupiter or high-order resonances were suspected, in which case the computationally more expensive integration method of Everhart (1974) was used. In both cases, the gravitational perturbations from all major planets were included.

From the result of the orbital integrations, the times at which the nodal distance was $1 \mathrm{AU}$ can be obtained, as can the values of all the orbital elements, which were taken to be the elements of the meteoroid stream. The values of the right ascension $\alpha$, declination $\delta$ of the radiant, and solar longitude $L_{\odot}$ of the postulated meteor shower were calculated as well as the geocentric velocity $V_{\mathrm{g}}\left(\mathrm{km} \mathrm{s}^{-1}\right)$.

A search of published catalogues and of the IAU Meteor Orbit Data Center (MODC) was then carried out to find real meteor events that matched the predicted ones. To quantify the similarity, the $D_{\mathrm{S}-\mathrm{H}}$ criterion of Southworth \& Hawkins (1963) was used. In addition, the positions of the predicted and observable radiant had to be closer than $\pm 10^{\circ}$ in both right ascension and declination, while the difference in geocentric velocity $\Delta V_{\mathrm{g}} \leq \pm 5 \mathrm{~km} \mathrm{~s}^{-1}$, and the period of activity to be within \pm 15 days of each other.

\section{Selected family of the NEAs that moved on similar comet-like orbits}

By applying the methodology described in the first two steps above, six potentially interesting NEAs were found, 2003HP2, 2006WX29, 2007VH189, 2007WT3, 2007WY3, and 2008UM1. In Table 1 the present-day orbital elements, Equinox 2000 as well as the nodal distances $R_{\mathrm{a}}$ and $R_{\mathrm{d}}$, the Tisserand invariant $T_{\mathrm{j}}$, the absolute magnitude $H$, and $d$, the equivalent diameter for all six NEAs are given. These were taken from the "Near-Earth Objects Dynamic Site"2.

The values of $T_{\mathrm{j}}$ indicate that all six NEAs are moving on comet-like orbits. Table 2 gives $D$ for all possible pairs. The highest value is 0.18 for the pair 2003HP32 and 2008UM1, all other values are considerably lower. In addition, $\pi$ lies in the range $333.7^{\circ} \pm 7.0^{\circ}$. These six thus have very similar orbits.

The equivalent diameters were calculated using the equation

$2 \log d=6.247-0.4 H-\log p$,

given by Tedesco et al. (1992), assuming a mean albedo $p$ of 0.07 . As can be seen in Table 1, the diameters range from $2 \mathrm{~m}$ to $1.1 \mathrm{~km}$ and are all too small to be the original comet nucleus, but the sizes are consistent with the being fragments.

\section{Orbital evolution of the NEAs}

The variations of the NEA orbital elements were calculated by the Halphen-Goryachev method with the exception of

2 http://newton.dm.unipi.it/neodys/ 
Table 2. Mutual values of the $D$ criterion of the NEAs.

\begin{tabular}{lcccccc}
\hline \hline NEA & 2003HP32 & 2006WX29 & 2007VH189 & 2007WT3 & 2007WY3 & 2008UM1 \\
\hline 2003HP32 & 0 & .06 & .13 & .08 & .06 & .18 \\
2006WX29 & .06 & 0 & .09 & .03 & .05 & .13 \\
2007VH189 & .13 & .09 & 0 & .06 & .08 & .05 \\
2007WT3 & .08 & .03 & .06 & 0 & .05 & .10 \\
2007WY3 & .06 & .05 & .08 & .05 & 0 & .12 \\
2008UM1 & .18 & .13 & .05 & .10 & .12 & 0 \\
\hline
\end{tabular}

Table 3. Theoretical $(\mathrm{T})$ and observed $(\mathrm{O})$ orbital elements, geocentric radiants, and velocities of meteor showers associated with the NEA 2007VH189.

\begin{tabular}{lccccccccccccc}
\hline \hline Showers & $q$ & $e$ & $i^{\circ}$ & $\Omega^{\circ}$ & $\omega^{\circ}$ & $L_{\odot}^{\circ}$ & Date & $\alpha^{\circ}$ & $\delta^{\circ}$ & $V_{\mathrm{g}}$ & $D_{\text {SH }}$ & Type & Catalogue \\
& & & & & & & & & & & & & \\
\hline $\mathrm{T}$ "AU) & .720 & .722 & 5.9 & 75.5 & 250.2 & 75.5 & June 6 & 245.1 & -11.1 & 19.0 & & $\mathrm{~N}$ & \\
$\mathrm{O} \chi$-Scords & .715 & .770 & 6.0 & 74.6 & 257.1 & 74.0 & June 5 & 247.7 & -13.1 & 21.0 & .09 & $\mathrm{~N}$ & $\mathrm{C}$ \\
\hline $\mathrm{T}$ "B" & .780 & .699 & 4.1 & 264.1 & 61.6 & 84.1 & June 14 & 245.0 & -29.9 & 16.9 & & $\mathrm{~N}$ & \\
$\mathrm{O} \delta$-Scords & .690 & .757 & .4 & 251.5 & 73.8 & 69.9 & May 31 & 239.7 & -21.1 & 20.2 & .13 & $\mathrm{~N}$ & $\mathrm{~T} 1$ \\
\hline $\mathrm{T}$ "C" & .780 & .703 & 4.3 & 208.6 & 117.1 & 208.6 & Oct. 21 & 223.3 & -7.6 & 17.4 & & $\mathrm{D}$ & \\
$\mathrm{O} \beta$-Librds & .757 & .533 & 3.1 & 217.3 & 104.2 & 217.3 & Oct. 30 & 223.9 & -9.5 & 18.0 & .18 & $\mathrm{D}$ & $\mathrm{MODC}$ \\
\hline $\mathrm{T}$ "D" & .830 & .681 & 4.4 & 20.0 & 305.7 & 200.0 & Oct. 13 & 214.4 & -24.3 & 15.6 & & $\mathrm{D}$ & \\
$\mathrm{O} \sigma$-Librds & .779 & .546 & 1.8 & 30.6 & 293.2 & 210.6 & Oct. 23 & 218.7 & -19.0 & 18.0 & .15 & $\mathrm{D}$ & $\mathrm{MODC}$ \\
\hline
\end{tabular}

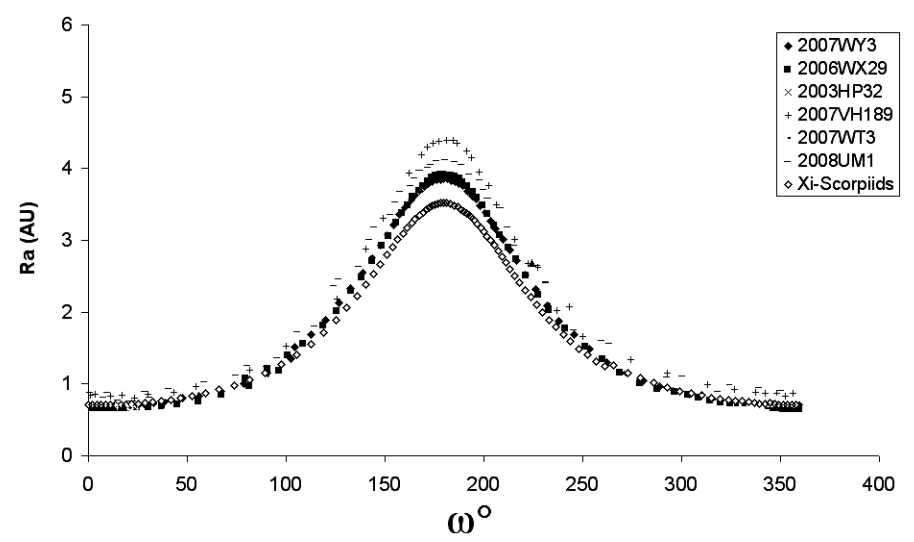

Fig. 1. Variations of the heliocentric distances of the ascending nodes $R_{\mathrm{a}}$ versus the arguments of perihelion $\omega$ of the six NEAs and the Scorpiids meteoroid stream.

2008UM1, where it was necessary to use the Everhart method because of resonances.

In Figs. 1 and 2 we plot the changes in the the heliocentric distances of the ascending and descending nodes $R_{\mathrm{a}}$ and $R_{\mathrm{d}}$ versus $\omega$ for each of the six NEAs. Interestingly, from the figures it can be seen that orbits of all NEAs have the values of $R_{\mathrm{a}} \approx 1 \mathrm{AU}$ when $\omega: 285.2 \pm 9.3^{\circ}$ and $75.3 \pm 7.9^{\circ}$, and $R_{\mathrm{d}} \approx 1 \mathrm{AU}$ at $104.6 \pm 8.5^{\circ}$ and $257.3 \pm 3.6^{\circ}$.

The orbital elements of the six NEAs at these four values of the argument of perihelion were taken as the orbits of the meteoroid stream that will produce the theoretical meteor showers.

\section{Meteor showers associated with the NEA family}

In Tables 3 and 4 the properties of the meteor showers that result from the meteoroid stream associated with two of the NEAs 2007VH189 and 2007WY3 are given as well as the orbital elements of the theoretical meteoroid streams. Following searches of meteor data bases of the observed meteor showers from Cook (1973) (C), Terentjeva (1966) (T1), and the IAU Meteor Orbit

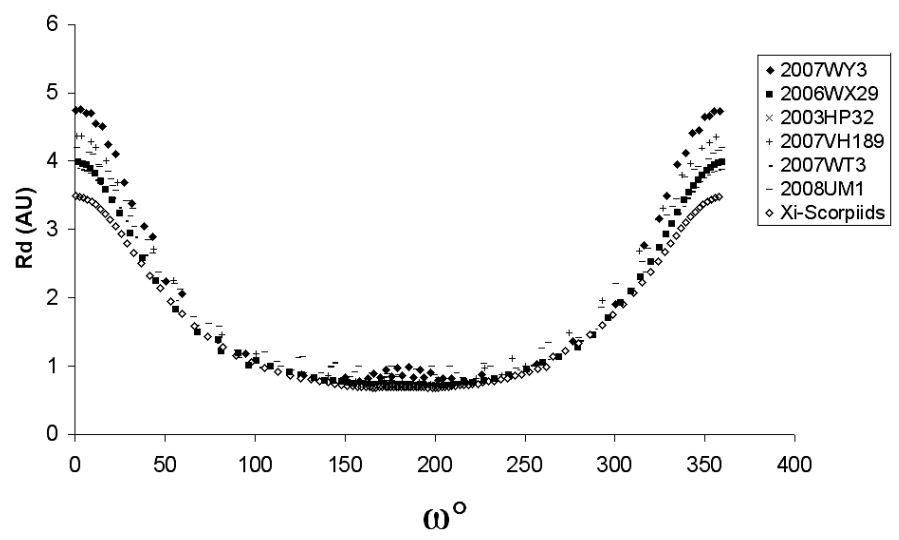

Fig. 2. Variations of the heliocentric distances of the descending nodes $R_{\mathrm{d}}$ versus the arguments of perihelion $\omega$ of the six NEAs and the Scorpiids meteoroid stream.

Data Center (MODC) found matching the theoretical predictions are also shown in the tables. The value of $D_{\mathrm{S}-\mathrm{H}}$ between the real and theoretical streams are also shown. Not surprisingly in view of the similarity between the orbits, the results for the remaining four streams are remarkably similar.

As seen from Tables 3 and 4 , all the theoretical showers have an observational match, with $D<0.15$ in all cases, the matches are the same showers. The strongest theoretical shower $\mathrm{T}^{\prime \prime} \mathrm{A}^{\prime \prime}$ (i.e. the last to form) is identified with the $\chi$-Scorpiids shower. The $\chi$-Scorpiids was first identified by Denning (1908), he observed several meteors emanating from $\alpha=252^{\circ}, \delta=-10^{\circ}$, during June $2-4$, and described them as "very slow". It has been regularly observed since that date. The $\delta$-Scorpiids meteor shower is the night-time counterpart of the daytime $\chi$-Scorpiids. It was first identified by Terentjeva (1966), giving the mean coordinates of radiant of the shower as $\alpha=239.7^{\circ}, \delta=-21.1^{\circ}$.

Because of the strong identification of the theoretical showers "A" with the meteoroid stream of the $\chi$-Scorpiids, the orbital evolution of the $\chi$-Scorpiids is also shown in Figs. 1 and 2. 
Table 4. Theoretical $(\mathrm{T})$ and observed $(\mathrm{O})$ orbital elements, geocentric radiants, and velocities of meteor showers associated with the NEA 2007WY3.

\begin{tabular}{|c|c|c|c|c|c|c|c|c|c|c|c|c|c|}
\hline Showers & $\begin{array}{c}q \\
(\mathrm{AU})\end{array}$ & $e$ & $i^{\circ}$ & $\Omega^{\circ}$ & $\omega^{\circ}$ & $L_{\odot}^{\circ}$ & Date & $\alpha^{\circ}$ & $\delta^{\circ}$ & $V_{\mathrm{g}}$ & $D_{\mathrm{SH}}$ & Type & Catalogue \\
\hline T "A" & .640 & .769 & 10.6 & 77.2 & 259.8 & 77.2 & June 8 & 253.7 & -7.4 & 22.2 & & $\mathrm{~N}$ & \\
\hline $\mathrm{O} \chi$-Scords & .715 & .770 & 6.0 & 74.6 & 257.1 & 74.0 & June 5 & 247.7 & -13.1 & 21.0 & .13 & $\mathrm{~N}$ & $\mathrm{C}$ \\
\hline T "B" & .660 & .763 & 8.0 & 259.8 & 77.2 & 79.8 & June 10 & 249.6 & -34.4 & 21.3 & & $\overline{\mathrm{N}}$ & \\
\hline $\mathrm{O} \delta$-Scords & .690 & .757 & .4 & 251.5 & 73.8 & 69.9 & May 31 & 239.7 & -21.1 & 20.2 & .11 & $\mathrm{~N}$ & $\mathrm{~T} 1$ \\
\hline $\mathrm{T} \quad$ "C" & .660 & .764 & 8.3 & 234.2 & 102.8 & 234.2 & Nov. 16 & 240.2 & -7.8 & 21.5 & & $\mathrm{D}$ & \\
\hline $\mathrm{O} \beta$-Librds & .689 & .633 & 6.5 & 230.9 & 104.4 & 230.9 & Nov. 12 & 238.2 & -8.6 & 21.9 & .14 & D & MODC \\
\hline T "D" & .650 & .768 & 10.8 & 56.2 & 280.8 & 236.2 & Nov. 18 & 234.0 & -35.6 & 22.3 & & $\mathrm{D}$ & \\
\hline O $\sigma$-Librds & .606 & .771 & 6.2 & 56.7 & 276.3 & 236.7 & Nov. 18 & 234.3 & -27.9 & 24.6 & .11 & D & MODC \\
\hline
\end{tabular}

For the predicted weaker showers that match the theoretical streams "C" and "D", 25 and 14 radar meteors were found using the database of the IAU MODC, respectively. These have not been named as meteor showers as yet. However, the mean radiants lie close to stars in Libra, and we propose that they be refereed to as the daytime $\beta$ - and $\sigma$-Librids.

\section{Conclusions}

We have found six NEAs that are moving on orbits that are close to each other and are comet-like. We have shown that meteoroid streams formed on these orbits will produce four meteor showers that correspond to observed showers, in particular the Scorpiid meteoroid stream. The orbital evolution of both the Scorpiids and the six NEAs remain nearly identical for several thousand years.

All these pointers lead us to the conclusion that several thousand years ago a comet nucleus fragmented, leaving six NEAs that should be regarded as cometary in nature, and a meteoroid stream that gives rise to four observable meteor showers, $\chi$ - and $\delta$-Scorpiids and the newly found $\beta$ - and $\sigma$-Librids

Acknowledgements. We would like to express our gratitude to the anonymous referee for useful comments which improved the paper. This work was supported by the International Science and Technology Center Project T-1629.

\section{References}

Asher, D. J., Clube, S. V. M., \& Steel, D. I. 1993, MNRAS, 264, 93

Asher, D. J., Bailey, M. E., \& Emel'Yanenko, V. V. 1999, MNRAS, 304, L53

Babadzhanov, P. B. 1996, Sol. Sys. Res., 30, 391

Babadzhanov, P. B. 2001, A\&A., 373, 329

Babadzhanov, P. B. 2003, A\&A., 397, 319

Babadzhanov, P. B., \& Obrubov, Yu. V. 1992, Cel. Mech. \& Dyn. Astron., 54, 111

Babadzhanov, P. B., \& Williams, I. P. 2007, in Near-Earth objects our celestial neighbors: opportunity and risk, eds. A. Milani, G. B. Valsecchi, \& D. Vokrouhlicky (Cambridge University Press), Proc. IAU Symp., 236, 135

Babadzhanov, P. B., Williams, I. P., \& Kokhirova, G. I. 2008a, MNRAS, 386, 1436

Babadzhanov, P. B., Williams, I. P., \& Kokhirova, G. I. 2008b, A\&A., 479, 249 Babadzhanov, P. B., Williams, I. P., \& Kokhirova, G. I. 2009, A\&A., 507, 1067 Babadzhanov, P. B., Williams, I. P., \& Kokhirova, G. I. 2012, MNRAS, 420, 2546

Cook, A. F. 1973, in Evolutionary and Physical Properties of Meteoroids, eds. C. L. Hemenway, P. M. Millman, \& A. F. Cook, NASA SP, 319 (Washington, DC), 183

Denning, W. F. 1908, The Observatory, 31, 287

Everhart, E. 1974, Celest. Mech., 10, 35

Farinella, P., Gonzi, R., Froeshle, Ch., \& Froeshle, Cl. 1992, in Lunar and Planetary Inst., Asteroids, Comets, Meteors 1991, eds. A. Harris, \& E. Bowell (Houston: LPI), 167
Fernandez, Y. R., Jewitt, D., \& Shepard, S. S. 2001, ApJ, 553, L197 Fox, K., Williams, I. P., \& Hughes, D. W. 1983, MNRAS, 205, 1155 Goryachev, N. N. 1937, Halphen's Method for Calculation of Planetary Secular Perturbations and its Application to Ceres, Krasnoe Znamya Tomsk

Hsieh, H. H., \& Jewitt, D. 2006, Science, 312, 561

Hughes, D. W. 1986, in Asteroids, Comets, Meteors II, eds. C.-I. Lagerkvist, B. A. Lindblad, H. Lundstedt, \& H. Rickman (Uppsala Universitet Reprocentralen), 503

Hunt, J., Williams, I. P., \& Fox, K. 1985, MNRAS, 217, 533

Jenniskens, P. 2004, AJ, 127, 3018

Jenniskens, P. 2006, Meteor Showers and Their Parent Comets (Cambridge, UK: Cambridge Univ. Press)

Jenniskens, P., \& Vaubaillon, J. 2007, AJ, 134, 1037

Jenniskens, P., \& Vaubaillon, J. 2008, AJ, 136, 725

Jewitt, D. C. 2012, AJ, 143, 66

Jewitt, D., \& Hsieh, H. 2006, AJ, 132, 1624

Jewitt, D., Weaver, H., Agarwal, J., Mutchler, M., \& Drahus, M. 2010, Nature, 467,817

Jopek, T., \& Williams, I. P. 2013, MNRAS, 430, 2377

Kasuga, T., \& Jewitt, D. 2008, AJ, 136, 881

Kinoshita, D., Ohtsuka, K., Sekiguchi, T., et al. 2007, A\&A, 466, 1153

Kresak, L. 1969, Bull. Astr. Inst. Czech-Sl., 20, 177

Lindblad, B. A. 1971a, Smithson. Contrib. Astrophys., 12, 1

Lindblad, B. A. 1971b, Smithson. Contrib. Astrophys., 12, 14

Madiedo, J. M., Trigo-Rodríguez, J. M., Williams, I. P., et al. 2013, MNRAS, 431,2464

Neslusan, L., Kanuchova, Z., \& Tomko, D. 2013, A\&A, 551, 87

Ohtsuka, K., Sekiguchi, T., Kinoshita, D., et al. 2006, A\&A, 450, L25

Olsson-Steel, D. 1988, Icarus, 75, 64

Porubčan, V., Kornos, L., \& Williams, I. P. 2004, Earth, Moon \& Planets, 95, 697

Porubčan, V., Kornos, L., \& Williams, I. P. 2006, Contrib. Astron. Obs. Scalnate Pleso, 36, 103

Ryabova, G. O. 2007, MNRAS, 375, 1371

Snodgrass, C., Tubiana, C., Vincent, J.-B., et al. 2010, Nature, 467, 814

Southworth, R. B., \& Hawkins, G. S. 1963, Smith. Contrib. Astrophys., 7, 261

Tedesco, E. F., Veeder, G. J., Fowler, J. W., \& Chillemi, J. R. 1992, in The IRAS Minor Planet Survey, Final Report, Philliips Laboratory, Hanscom Air Force Base, MA, PL-TR-92-2049

Terentjeva, A. K. 1966, in Results of investigations on international geophysical projects, Investigations of meteors (Moscow: Nauka), 62

Trigo-Rodriguez, J. M., Lyytinen, E., Jones, D. C., et al. 2007, MNRAS, 382, 1933

Trigo-Rodriguez, J. M., Madiedo, J. M., Williams, I. P., \& Castro-Tirado, A. J. 2008a, MNRAS, 392, 367

Trigo-Rodriguez, J. M., Madiedo, J. M., Williams, I. P., et al. 2008b, MNRAS, 394, 569

Whipple, F. L. 1951, ApJ, 113, 464

Whipple, F. L. 1983, IAU Circ., 3881

Williams, I. P. 1993, in Meteoroids and their parent bodies, eds. J. Štohl, \& I. P. Williams, Slovak Acad. Sci., Bratislava, 31

Williams, I. P. 1995, Earth, Moon \& Planets, 68,

Williams, I. P. 2011, Astron. Geophys., 52, 220

Williams, I. P., \& Wu, Z. 1993, MNRAS, 262, 231

Williams, I. P., Ryabova, G. O., Baturin, A. P., \& Chernitsov, A. M. 2004 MNRAS, 355, 1171

Wu, Z., \& Williams, I. P. 1992, MNRAS, 259, 617 